\title{
THE DETERMINANT OF THE EISENSTEIN MATRIX AND HILBERT CLASS FIELDS
}

\author{
BY
}

I. EFRAT AND P. SARNAK ${ }^{1}$

\begin{abstract}
We compute the determinant of the Eisenstein matrix associated to the Hilbert-Blumenthal modular group $\operatorname{PSL}_{2}\left(O_{k}\right)$, and express it in terms of the zeta function of the Hilbert class field of $K$.
\end{abstract}

0. Introduction. The Eisenstein series for the modular group $\Gamma=\mathrm{PSL}_{2}(\mathbf{Z})$ is given by

$$
E(z, s)=\sum_{\gamma \in \Gamma_{\infty} \backslash \Gamma} y(\gamma z)^{s}
$$

where $\Gamma_{\infty}=\left\{\left(\begin{array}{ll}1 & n \\ 0 & 1\end{array}\right) \mid n \in \mathbf{Z}\right\}, z$ is in the upper half-plane and $y(z)=\operatorname{Im}(z)$. The Fourier expansion of $E(z, s)$ is well known (see Hejhal $[4$, p. 76]):

$$
E(z, s)=y^{s}+\phi(s) y^{1-s}+\text { nonzero coefficients, }
$$

where

$$
\phi(s)=\frac{\pi^{-(s-1 / 2)} \Gamma(s-1 / 2) \varsigma(2 s-1)}{\pi^{-s} \Gamma(s) \zeta(2 s)},
$$

$\Gamma(s)$ is the gamma function and $\zeta(s)$ is the Riemann zeta function.

In the general case of a group with $h$ cusps one has an Eisenstein series at each cusp. The Fourier expansions of these series in the various cusps lead to an $h \times h$ matrix $\Phi(s)$ (see (1.11) below). This matrix is the constant term of the Eisenstein series, and is sometimes referred to as the scattering matrix [7], or simply the $\Phi$-matrix.

The determinant $\phi(s)=\operatorname{det} \Phi(s)$ plays an important role in the theory. In the first place it controls the Eisenstein series in the sense that if $\phi(s)$ is analytic at some point, then so are all the Eisenstein series. More importantly, $\phi(s)$ (or rather its logarithmic derivative) accounts for the contribution of the continuous spectrum to the Selberg Trace Formula. A classical situation in which multiplicity of cusps occurs is that of congruence subgroups of the modular group, and the computation of $\phi(s)$ for these is quite involved (see Hejhal [4, Chapter 12] and Huxley [5]).

Our aim in this paper is to calculate $\phi(s)$ for the Hilbert-Blumenthal modular groups $\mathrm{PSL}_{2}\left(O_{K}\right), O_{K}$ being the ring of integers of a number field $K$. In this case the number of cusps is the class number of $K$. What we show is that $\phi(s)$ is elegantly expressed in terms of the zeta function of the Hilbert class field of $K$. An interesting feature of our approach is that we do not use any Fourier developments

Received by the editors October 3, 1984.

1980 Mathematics Subject Classification. Primary 10D15, 10D21; Secondary 10D40, 12A65, $12 \mathrm{~A} 70$.

1 The work of the second author was supported by NSF Grant MCS-82-01599 and a Sloan Fellowship and the first autinor by NSF Grant DMS-84-02740.

(C) 1985 American Mathematical Society $0002-9947 / 85 \$ 1.00+\$ .25$ per page 
in cusps. Instead we obtain our result purely from considerations of functional equations. To keep notations to a minimum we shall deal only with imaginary quadratic fields. The general case may be dealt with in a similar way (see Efrat [2] for the set up for totally real fields).

1. General set up and results. Let $\mathfrak{h}^{3}$ be the hyperbolic three space $\{w=$ $\left.\left(y, x_{1}, x_{2}\right)=(y, z) \mid y>0\right\}$ with its Lobachevskii metric

$$
d s^{2}=\frac{d y^{2}+d x_{1}^{2}+d x_{2}^{2}}{y^{2}}
$$

If we think of $\mathfrak{h}^{3}$ as the set of all quaternions $x_{1}+i x_{2}+j y+k t$ for which $t=0$, then $G=\mathrm{PSL}_{2}(\mathbf{C})$ is the group of all orientations preserving isometries of $\mathfrak{h}^{3}$. It acts via linear fractional transformations, i.e., for $\tau=\left(\begin{array}{ll}\alpha & \beta \\ \gamma & \delta\end{array}\right) \in G$,

$$
\tau(w)=(\alpha w+\beta)(\gamma w+\delta)^{-1} .
$$

Consider next a discrete subgroup $\Gamma \subset G$ for which $₹=\mathfrak{h} / \Gamma$ is not compact but is of finite volume. Let $\kappa_{1}=\infty, \kappa_{2}, \ldots, \kappa_{n}$ be a complete set of inequivalent cusps, and let $\Gamma_{i}$ be the subgroup of $\Gamma$ that fixes $\kappa_{i}$. We choose $\rho_{i} \in G$ such that $\rho_{i}\left(\kappa_{i}\right)=\infty$ and such that

$$
\rho_{i} \Gamma_{i} \rho_{i}^{-1}=\left\{\left(\begin{array}{ll}
1 & l \\
0 & 1
\end{array}\right) \mid l \in L_{i}\right\}
$$

where $L_{i}$ is a lattice in $\mathbf{C}$ whose fundamental domain has volume 1 . We let

$$
w^{(i)}=\rho_{i} w=\left(y\left(\rho_{i} w\right), z\left(\rho_{i} w\right)\right) .
$$

DEFINITION 1.1. For each cusp $\kappa_{i}$, define its Eisenstein series to be

$$
E_{i}(w, s)=\sum_{\gamma \in \Gamma_{i} \backslash \Gamma} y^{(i)}(\gamma w)^{s}, \quad \operatorname{Re}(s)>2 .
$$

Then Selberg's theory shows that $E_{i}(w, s)$ can be meromorphically continued in $s$ to all of $\mathbf{C}$, and gives a $\Gamma$-automorphic eigenfunction of the Laplacian $\Delta$ of $\mathfrak{h}^{3}$, with

$$
\Delta E_{i}(w, s)+s(2-s) E_{i}(w, s)=0 .
$$

Since $E_{i}(w, s)$ is $\Gamma$-automorphic, it is invariant under the lattice at $\kappa_{j}$. It thus admits a Fourier expansion there, which is of the form

$$
\begin{aligned}
E_{i}(w, s)= & \delta_{i j} y^{(j)^{s}}+\phi_{i j}(s) y^{(j)^{2-s}} \\
& + \text { nonzero coefficients, rapidly decaying as } y^{(j)} \rightarrow \infty .
\end{aligned}
$$

If we let $\Phi(s)=\left(\phi_{i j}(s)\right)_{i, j=1, \ldots, h}$ and

$$
\vec{E}(w, s)=\left[\begin{array}{c}
E_{1}(w, s) \\
\vdots \\
E_{h}(w, s)
\end{array}\right],
$$

then we have the functional equation

$$
\vec{E}(w, s)=\Phi(s) \vec{E}(w, 2-s)
$$

(For these facts see Cohen and Sarnak [1], Hejhal [4] or Sarnak [8].) The following lemma shows that $\Phi(s)$ is characterized by this equation. 
LEMMA 1.2. If $\vec{E}(w, s)=\Psi(s) \vec{E}(w, 2-s)$ for every $w \in \mathfrak{h}^{3}$, then $\Psi(s)=\Phi(s)$.

Proof. We have

$$
\begin{aligned}
E_{i}(w, s) & =\sum_{k=1}^{h} \psi_{i k}(s) E_{k}(w, 2-s) \\
& =\sum_{k=1}^{h} \psi_{i k}(s)\left(\delta_{k j} y^{(j)^{2-s}}+\phi_{k j}(2-s) y^{(j)^{s}}+\cdots\right) .
\end{aligned}
$$

Now choose $s$ with $\operatorname{Re}(s)<0$. Then as $y^{(j)} \rightarrow \infty$ only the terms involving $y^{(j)^{2-s}}$ will increase. Comparing (1.2.1) with (1.1.1) gives

$$
\phi_{i j}(s)=\sum_{k=1}^{h} \psi_{i k} \delta_{k j}=\psi_{i j}(s)
$$

Let $\Gamma=\Gamma_{D}$ be the Hilbert modular group associated to the imaginary quadratic number field $K=Q(\sqrt{-D})$, i.e.,

$$
\Gamma_{D}=\mathrm{PSL}_{2}\left(O_{K}\right)=\left\{\left(\begin{array}{ll}
a & b \\
c & d
\end{array}\right) \mid a, b, c, d \in O_{K}, a d-b c=1\right\} /\{ \pm 1\},
$$

where $O_{K}$ is the ring of integers of $K$. We also assume $D \neq 1,3$.

Then the cusps of $\Gamma$ are the numbers $\alpha / \beta \in \mathbf{C}$ with $\alpha, \beta \in O_{K}$ and $\infty$. Furthermore, $\alpha / \beta$ and $\alpha^{\prime} / \beta^{\prime}$ are equivalent under $\Gamma$ as cusps if and only if $(\alpha, \beta)$ and $\left(\alpha^{\prime}, \beta^{\prime}\right)$ are equivalent ideals. So if $A_{1}, \ldots, A_{h}, A_{i}=\left(\gamma_{i}, \delta_{i}\right)$, are a compelte set of representatives of the ideal classes of $K$, then $\kappa_{i}=-\delta_{i} / \gamma_{i}, i=1, \ldots, h$, form a complete set of inequivalent cusps.

To define the $\rho_{i}$ 's we first note that we can choose $\alpha_{i}, \beta_{i} \in \mathcal{A}_{i}^{-1}$ such that

$$
\hat{\rho}_{i}=\left(\begin{array}{cc}
\alpha_{i} & \beta_{i} \\
\gamma_{i} & \delta_{i}
\end{array}\right) \in G
$$

Note that $\hat{\rho}_{i}\left(\kappa_{i}\right)=\infty$.

To guarantee the volume 1 condition, we scale $\hat{\rho}_{i}$ and define

$$
\rho_{i}=\left(\begin{array}{cc}
\lambda_{i}^{1 / 2} & 0 \\
0 & \lambda_{i}^{-1 / 2}
\end{array}\right) \hat{\rho}_{i}
$$

where $\lambda_{i}=\omega_{K} N A_{i}, \omega_{K}=\sqrt{2} / d_{K}^{1 / 4}$ and $d_{K}$ is the absolute value of the discriminant of $K$.

By the discussion above, we now have our Eisenstein series defined for $\Gamma_{D}$. Our main theorem is

THEOREM 1. For $\Gamma_{D}$,

$$
\phi(s)=(-1)^{\left(h-2^{t-1}\right) / 2} \omega_{K}^{2 s-2} \frac{\xi_{H}(s-1)}{\xi_{H}(s)},
$$

where $\xi_{H}(s)=\left(d_{H}^{1 / 2} /(2 \pi)^{h}\right)^{s} \Gamma(s)^{h} \zeta_{H}(s), H$ is the Hilbert class field of $K$ and $t$ is the number of prime divisors of $d_{K}$.

REMARKS. (i) Since $H$ is unramified everywhere it follows that $\xi_{H}(s)$ is the usual $\xi$ function for the Hilbert class field $H$, so that $\xi_{H}(1-s)=\xi_{H}(s)$. 
(ii) The exponent $\left(h-2^{t-1}\right) / 2$ of -1 in the formula is always an integer by genus theory (see, for example, Hecke [3, p. 160]).

Besides the contribution $\phi(s)$ to the trace formula there is also one other term that appears and which comes from the Eisenstein series. It is the term $\operatorname{tr}(\Phi(1))$ (see [1 or 4]).

Since $\Phi(s)$ is real symmetric for real $s$ and is unitary for $s$ of the form $1+i t$, it follows that at this special point (the middle of the critical strip) $\Phi(1)$ is both unitary and real symmetric. Its eigenvalues are therefore \pm 1 , so that the trace is an integer which counts the excess of the eigenvalue +1 over -1 (or to put it another way we want the signature of $\Phi(1))$. We have

THEOREM 2. $\operatorname{tr}(\Phi(1))=2^{t-1}-2$, where, as above, $t$ is the number of prime divisors of $d_{K}$.

One final comment before turning to the proofs of these theorems: In the general number field case the formulas of Theorems 1 and 2 are similar, however the number $2^{t-1}$ is replaced by the number of elements of order two in the class group. In the quadratic case this number may be determined in terms of the divisors of $d$ as we have done.

2. Functional equations and the matrix $\Phi(s)$. We begin this section by deriving the functional equation for the Eisenstein series using theta functions. A nice discussion of Eisenstein series and Epstein zeta functions appears in Terras $[\mathbf{9}$, especially Chapter 5]. Since these functional equations are central to the approach in this paper we derive them directly using the notation we have introduced.

For a quaternion $w=x_{1}+i x_{2}+j y+k t$ we denote by $N(w)$ its norm: $N(w)=$ $x_{1}^{2}+x_{2}^{2}+y^{2}+t^{2}$. Its fundamental property is $N\left(w_{1} w_{2}\right)=N\left(w_{1}\right) N\left(w_{2}\right)$. If $w \in \mathfrak{h}^{3}$ and $y(w)$ is its $y$-coordinate, then for $\tau \in \mathrm{PSL}_{2}(\mathbf{C}), \tau=\left(\begin{array}{ll}\alpha & \beta \\ \gamma & \delta\end{array}\right)$, we have $y(\tau w)=y / N(\gamma w+\delta)$.

DEFINITION 2.1. Define $\tilde{E}_{i}(w, s)$ by

$$
\tilde{E}_{i}(w, s)=\lambda_{i}^{s} \sum_{(c, d)=A_{i}} \frac{y^{s}}{N(c w+d)^{s}} .
$$

We will need the following, by now standard, lemma due to Selberg.

LEMMA 2.2. Let $f(w)$ be a $\gamma$-automorphic function on $\mathfrak{h}^{3}$ satisfying

$$
\Delta f+s(2-s) f=0
$$

for some $s$ with $\operatorname{Re}(s)>2$ and such that

$$
f(w)=\delta_{i j}\left(y^{(j)}\right)^{s}+O(1) \quad \text { as } y^{(j)} \rightarrow \infty .
$$

Then $f(w)=E_{i}(w, s)$.

PROOF. $H_{i}(w)=E_{i}(w, s)-f(w)$ is a $\Gamma$-automorphic eigenfunction of $\Delta$ with eigenvalue $s(2-s)$. In view of the behaviour of $f(w)$ in the cusps, $H_{i}$ is in $L^{2}\left(\Gamma \backslash \mathfrak{h}^{3}\right)$. However, from the selfadjointness and nonnegativity of $\Delta$ it follows that its eigenvalue must be real and nonnegative. For $s(2-s)$ with $\operatorname{Re}(s)>2$ this is not the case, so $H_{i} \equiv 0$. 
Proposition 2.3. $\tilde{E}_{i}(w, s)=E_{i}(w, s)$.

ProOF. We verify the conditions of Lemma 2.2 for $\tilde{E}_{i}$. First, $\tilde{E}_{i}(w, s)$ is $\Gamma$ automorphic, because if $\gamma=\left(\begin{array}{ll}x & y \\ u & v\end{array}\right) \in \Gamma$, then

$$
\begin{aligned}
\tilde{E}_{i}(\gamma w, s) & =\lambda_{i}^{s} \sum_{(c, d)=A_{i}} \frac{y^{s} / N(u w+v)^{s}}{N\left(c(x w+y)(u w+v)^{-1}+d\right)^{s}} \\
& =\lambda_{i}^{s} \sum_{(c, d)=A_{i}} \frac{y^{s} / N(u w+v)^{s}}{N(c(x w+y)+d(u w+v))^{s} N(u w+v)^{-s}} \\
& =\lambda_{i}^{s} \sum_{(c, d)=A_{i}} \frac{y^{s}}{N((c x+d u) w+(c y+d v))^{s}}
\end{aligned}
$$

and $c x+d u$ and $c y+d v$ run over the basis of $A_{i}$ as $c$ and $d$ do.

To check the behaviour of $\tilde{E}_{i}$ at the $j$ th cusp, recall that

so that

$$
\rho_{j}^{-1}=\left(\begin{array}{cc}
\delta_{j} & -\beta_{j} \\
-\gamma_{j} & \alpha_{j}
\end{array}\right)\left(\begin{array}{cc}
\lambda_{j}^{-1 / 2} & 0 \\
0 & \lambda_{j}^{1 / 2}
\end{array}\right)
$$

$$
\begin{aligned}
& \tilde{E}_{i}(w, s)=\tilde{E}_{i}\left(\rho_{j}^{-1} w^{(j)}, s\right)=\lambda_{i}^{s} \sum_{(c, d)=\mathcal{A}_{i}} \frac{y\left(\rho_{j}^{-1} w^{(j)}\right)^{s}}{N\left(c \rho_{j}^{-1} w^{(j)}+d\right)^{s}} \\
& =\lambda_{i}^{s} \sum_{(c, d)=A_{i}} \frac{y^{(j)^{s}} / N\left(-\gamma_{j} \lambda_{j}^{-1 / 2} w^{(j)}+\alpha_{j} \lambda_{j}^{1 / 2}\right)^{s}}{N\left(c\left(\delta_{j} \lambda_{j}^{-1 / 2} w^{(j)}-\beta_{j} \lambda_{j}^{1 / 2}\right)\left(-\gamma_{j} \lambda_{j}^{-1 / 2} w^{(j)}+\alpha_{j} \lambda_{j}^{1 / 2}\right)^{-1}+d\right)^{s}} \\
& =\lambda_{i}^{s} \sum_{(c, d)=A_{i}} \frac{y^{(j)^{s}}}{N\left(\left(c \delta_{j} \lambda_{j}^{-1 / 2}-d \gamma_{j} \lambda_{j}^{-1 / 2}\right) w^{(j)}+\left(-c \beta_{j} \lambda_{j}^{1 / 2}+d \alpha_{j} \lambda_{j}^{1 / 2}\right)\right)^{s}} \\
& =\frac{\lambda_{i}^{s}}{\lambda_{j}^{s}} \sum_{(c, d)=A_{i}} \frac{y^{(j)^{s}}}{N\left(\lambda_{j}^{-1}\left(c \delta_{j}-d \gamma_{j}\right) w^{(j)}+\left(-c \beta_{j}+d \alpha_{j}\right)\right)^{s}} .
\end{aligned}
$$

The only term in this sum which grows with $y^{(j)}$ is the one for which $c \delta_{j}-$ $d \gamma_{j}=0,-c \beta_{j}+d \alpha_{j}=1$, which occurs if and only if $i=j$, so that the above is $\delta_{i j}\left[y^{(j)^{s}}+O(1)\right]$. Since $\tilde{E}_{i}$ is clearly an eigenfunction with eigenvalue $s(2-s)$, Lemma 2.2 implies our claim.

DEFINITION 2.4.

$$
F_{i}(w, s)=\lambda_{i}^{s} \sum_{\substack{c, d \in A_{i} \\(\bmod \pm 1)}}^{\prime} \frac{y^{s}}{N(c w+d)^{s}}
$$

(so that we run over half the lattice $A_{i} \times A_{i}$ ).

Proposition 2.5.

$$
\vec{F}(w, s)=\left[\begin{array}{c}
F_{1}(w, s) \\
\vdots \\
F_{h}(w, s)
\end{array}\right]=\left[\varsigma_{\mathcal{A}_{i}^{-1} \mathcal{A}_{j}}(s)\right]\left[\begin{array}{c}
E_{1}(w, s) \\
\vdots \\
E_{h}(w, s)
\end{array}\right],
$$

where $\zeta_{A}(s)$ is the Dedekind zeta function of the ideal class of $A$. 
Proof (SEE TERras $[\mathbf{9}]$ ).

$$
\begin{aligned}
F_{i}(w, s) & =\lambda_{i}^{s} \sum_{\mathcal{A}_{i} \mid B} \sum_{(c, d)=B} \frac{y^{s}}{N(c w+d)^{s}} . \\
& =\lambda_{i}^{s} \sum_{j=1}^{h} \sum_{\substack{A_{i} \mid B \\
B \sim A_{j}}} \sum_{(c, d)=B} \frac{y^{s}}{N(c w+d)^{s}} .
\end{aligned}
$$

Write $B=\left(\theta_{B}\right) A_{j}$ for some $\theta_{B} \in K$. Then

$$
\begin{aligned}
& \lambda_{i}^{s} \sum_{j=1}^{h} \sum_{\substack{\mathcal{A}_{i} \mid \mathrm{B} \\
B \sim \mathcal{A}_{j}}} \frac{1}{N \theta_{B}^{s}} \sum_{\left(c / \theta_{B}, d / \theta_{B}\right)=\mathcal{A}_{j}} \frac{y^{s}}{N\left(c w / \theta_{B}+d / \theta_{B}\right)^{s}} \\
& =\lambda_{i}^{s} \sum_{j=1}^{h}\left(\sum_{\substack{A_{i} \mid B \\
B \sim A_{j}}} \frac{1}{N B^{s}}\right) N A_{j}^{s} \sum_{(c, d)=A_{j}} \frac{y^{s}}{N(c w+d)^{s}} .
\end{aligned}
$$

Now let $C=A_{i}^{-1} B$ so that $C \sim A_{i}^{-1} A_{j}, N B=N C \cdot N A_{i}$; then

$$
=\sum_{j=1}^{h}\left(\sum_{C \sim \mathcal{A}_{i}^{-1} \mathcal{A}_{j}} \frac{1}{N C^{s}}\right) \lambda_{j}^{s} \sum_{(c, d)=\mathcal{A}_{j}} \frac{y^{s}}{N(c w+d)^{s}} .
$$

PROPOSITION 2.6. The function $\omega_{K}^{-s}\left(d_{K}^{1 / 2} / 2 \pi\right)^{s} \Gamma(s) F_{i}(w, s)$ is invariant under $s \rightarrow 2-s$ and $A_{i} \rightarrow\left(\partial_{K} A_{i}\right)^{-1}$, where $\partial_{K}$ is the different of $K$.

Proof. Using the formula

$$
\frac{\Gamma(s)}{a^{s}}=\int_{0}^{\infty} e^{-a t} t^{s} \frac{d t}{t}
$$

with $a=2 \pi / d_{K}^{1 / 2} N A_{i} \cdot N(c w+d) / y$, we get

$$
\begin{aligned}
\omega_{K}^{-s} & \cdot\left(\frac{d_{K}^{1 / 2}}{2 \pi}\right)^{s} \Gamma(s)\left(\omega_{K} N \mathcal{A}_{i}\right)^{s} \sum_{c, d \in \mathcal{A}_{i}}^{\prime} \frac{y^{s}}{N(c w+d)^{s}} \\
& =\int_{0}^{\infty} \sum_{c, d \in A_{i}}^{\prime} \exp \left(-\frac{2 \pi}{d_{K}^{1 / 2} N A_{i}} \frac{N(c w+d)}{y} t\right) t^{s} \frac{d t}{t} \\
& =\int_{0}^{\infty} \sum_{l \in \mathcal{A}_{i} \times A_{i}}^{\prime} \exp \left(-\frac{2 \pi}{d_{K}^{1 / 2} N A_{i}}\langle l, A l\rangle t\right) t^{s} \frac{d t}{t}
\end{aligned}
$$

where $A$ is the Hermitian matrix

$$
A=\left(\begin{array}{cc}
N w / y & z / y \\
\bar{z} / y & 1 / y
\end{array}\right)
$$


We wish to apply the Poisson summation formula to this last expression. To this end, let

$$
f\left(z_{1}, z_{2}\right)=e^{-r\langle Z, A Z\rangle}, \quad Z=\left(\begin{array}{c}
z_{1} \\
z_{2}
\end{array}\right),
$$

be thought of as a function of four real variables. Then for $(\beta, \gamma) \in \mathbf{C}^{2}$,

$$
\hat{f}(\beta, \gamma)=\iint_{\mathbf{C}^{2}} f\left(z_{1}, z_{2}\right) e^{-2 \pi i \operatorname{Re}\left(\bar{\beta} z_{1}+\bar{\gamma} z_{2}\right)} d Z
$$

where $d Z=-\frac{1}{4} \cdot d z_{1} \wedge \overline{d z_{1}} \cdot d z_{2} \wedge \overline{d z_{2}}$,

$$
=\iint_{\mathbf{C}^{2}} e^{-r\langle Z, A Z\rangle} e^{-2 \pi i \operatorname{Re}\langle(\beta, \gamma), Z\rangle} d Z .
$$

Since $A$ is positive definite we can change variables $W=A^{1 / 2} Z$. Since $|A|=1$ this leads to

$$
\iint_{\mathbf{C}^{2}} e^{-r\langle W, W\rangle} e^{-2 \pi i \operatorname{Re}\left\langle A^{-1 / 2}\left(\begin{array}{l}
\beta \\
\gamma
\end{array}\right), W\right\rangle} d W
$$

Writing

$$
W=\left[\begin{array}{l}
u_{1} \\
u_{2} \\
v_{1} \\
v_{2}
\end{array}\right] \quad \text { and } \quad A^{-1 / 2}\left(\begin{array}{c}
\beta \\
\gamma
\end{array}\right)=\left[\begin{array}{c}
\mu_{1} \\
\mu_{2} \\
\nu_{1} \\
\nu_{2}
\end{array}\right]
$$

we have

$$
\int \ldots \mathbf{R}^{4} \int e^{-r\left(u_{1}^{2}+u_{2}^{2}+v_{1}^{2}+v_{2}^{2}\right)} e^{-2 \pi i\left(\mu_{1} v_{1}+\mu_{2} u_{2}+\nu_{1} v_{1}+\nu_{2} v_{2}\right)} d u_{1} d u_{2} d v_{1} d v_{2} .
$$

Recalling that

$$
\int_{-\infty}^{\infty} e^{-r x^{2}} e^{-2 \pi i x y} d x=\sqrt{\frac{\pi}{r}} e^{-\pi^{2} y^{2} / r}
$$

we obtain

$$
\begin{aligned}
& \frac{\pi^{2}}{r^{2}} e^{-\pi^{2}\left(\mu_{1}^{2}+\mu_{2}^{2}+\nu_{1}^{2}+\nu_{2}^{2}\right) / r} \\
& =\frac{\pi^{2}}{r^{2}} e^{-\pi^{2}\left\langle A^{-1 / 2}\left({ }_{\gamma}^{\beta}\right), A^{-1 / 2}\left({ }_{\gamma}^{\beta}\right)\right\rangle / r} \\
& =\frac{\pi^{2}}{r^{2}} e^{-\pi^{2}\left\langle\left({ }_{\gamma}^{\beta}\right), A^{-1}\left({ }_{\gamma}^{\beta}\right)\right\rangle / r} .
\end{aligned}
$$

Going back to (2.6.1), we separate the integral into two parts to obtain

$$
\begin{aligned}
\int_{1}^{\infty} & \sum_{l \in A_{i} \times A_{i}}^{\prime} \exp \left(-\frac{2 \pi}{d_{K}^{1 / 2} N A_{i}}\langle l, A l\rangle t\right) t^{s} \frac{d t}{t} \\
& +\int_{0}^{1} \sum_{l \in A_{i} \times A_{i}} \exp \left(-\frac{2 \pi}{d_{K}^{1 / 2} N A_{i}}\langle l, A l\rangle t\right) t^{s} \frac{d t}{t}-\frac{1}{s} \\
& =\mathrm{I}+\mathrm{II} .
\end{aligned}
$$


Applying the Poisson summation formula to II we get from our computation above

$$
\mathrm{II}=\frac{1}{v} \int_{0}^{1} \frac{\pi^{2}}{r^{2}} \sum_{l \in \mathcal{A}_{i}^{\prime} \times \mathcal{A}_{i}^{\prime}} \exp \left(-\frac{\pi^{2}}{r}\left\langle l, A^{-1} l\right\rangle\right) t^{s} \frac{d t}{t}-\frac{1}{s},
$$

where $r=2 \pi t / d_{K}^{1 / 2} N A_{i}$ and $A_{i}^{\prime}$ is the dual lattice of $A_{i}$, so that $A_{i}^{\prime}=2\left(\partial_{K} A_{i}\right)^{-1}$. Here $v=\operatorname{vol}\left(\mathbf{C}^{2} / A_{i} \times A_{i}\right)=d_{K} / 4 \cdot N A_{i}^{2}$. Thus

$$
\mathrm{II}=\int_{0}^{1} \sum_{l \in\left(\partial_{K} \mathcal{A}_{i}\right)^{-1} \times\left(\partial_{K} A_{i}\right)^{-1}} \exp \left(-\frac{\pi d_{K}^{1 / 2} N A_{i}}{2}\left\langle 2 l, 2 A^{-1} l\right\rangle t^{-1}\right) t^{s-2} \frac{d t}{t}-\frac{1}{s} .
$$

Now change variables $t \rightarrow t^{-1}$

$$
\begin{aligned}
& =\int_{1}^{\infty} \sum_{l \in\left(\partial_{K} A_{i}\right)^{-1} \times\left(\partial_{K} A_{i}\right)^{-1}} \exp \left(-2 \pi d_{K}^{1 / 2} N A_{i}\left\langle l, A^{-1} l\right\rangle t\right) t^{2-s} \frac{d t}{t}-\frac{1}{s} \\
& =\int_{1}^{\infty} \sum_{c, d \in\left(\partial_{K} A_{i}\right)^{-1}}^{\prime} \exp \left(-2 \pi d_{K}^{1 / 2} N A_{i} \frac{N(c w+d)}{y} t\right) t^{2-s} \frac{d t}{t}-\frac{1}{s}-\frac{1}{2-s} .
\end{aligned}
$$

Finally we note that

$$
d_{K}^{1 / 2} N A_{i}=\frac{1}{d_{K}^{1 / 2} N\left(A_{i} \partial_{K}\right)^{-1}}
$$

and comparing our last expression with (2.6.1) completes the proof.

COROLlaRY 2.7. Let $P$ be the permutation matrix of the permutation of the ideal class group given by $A_{i} \rightarrow\left(\partial_{K} A_{i}\right)^{-1}$. Then

$$
\vec{F}(w, s)=\omega_{K}^{2 s-2} \frac{\left(d_{K}^{1 / 2} / 2 \pi\right)^{2-s} \Gamma(2-s)}{\left(d_{K}^{1 / 2} / 2 \pi\right)^{s} \Gamma(s)} \cdot P \cdot \vec{F}(w, 2-s) .
$$

Putting Proposition 2.5 and Corollary 2.7 together we obtain

$$
\left[\zeta_{\mathcal{A}_{i}^{-1} \mathcal{A}_{j}}(s)\right] \vec{E}(w, s)=\omega_{K}^{2 s-2} \frac{\left(d_{K}^{1 / 2} / 2 \pi\right)^{2-s} \Gamma(2-s)}{\left(d_{K}^{1 / 2} / 2 \pi\right)^{s} \Gamma(s)} \cdot P \cdot\left[\zeta_{\mathcal{A}_{i}^{-1} \mathcal{A}_{j}}(2-s)\right] \vec{E}(w, 2-s) .
$$

But then Lemma 1.2 implies

COROllary 2.8 .

$$
\Phi(s)=\omega_{K}^{2 s-2} \frac{\left(d_{K}^{1 / 2} / 2 \pi\right)^{2-s} \Gamma(2-s)}{\left(d_{K}^{1 / 2} / 2 \pi\right)^{s} \Gamma(s)}\left[\varsigma_{\mathcal{A}_{i}^{-1} \mathcal{A}_{j}}(s)\right]^{-1} \cdot P \cdot\left[\zeta_{\mathcal{A}_{i}^{-1} \mathcal{A}_{j}}(2-s)\right] .
$$

3. Computations of $\phi(s)$ and $\operatorname{tr} \Phi(1)$. We can finally turn to the computation of $\phi(s)$. We first find $\operatorname{det}\left[\varsigma_{\mathcal{A}_{i}^{-1}} \mathcal{A}_{j}(s)\right]$.

PROPOSITION 3.1. Let $\chi$ be a character of the ideal class group and let $L(s, \chi)$ be its L-function. Then

$$
\left[\varsigma_{\mathcal{A}_{i}^{-1} A_{j}}(s)\right]\left[\begin{array}{c}
\chi\left(A_{1}\right) \\
\vdots \\
\chi\left(A_{h}\right)
\end{array}\right]=L(s, \chi)\left[\begin{array}{c}
\chi\left(A_{1}\right) \\
\vdots \\
\chi\left(A_{h}\right)
\end{array}\right] .
$$


PROOF.

$$
\begin{aligned}
\sum_{j=1}^{h} S_{A_{i}^{-1} A_{j}}(s) \cdot \chi\left(A_{j}\right) & =\sum_{j=1}^{h} \sum_{B \sim A_{i}^{-1} A_{j}} \frac{\chi\left(A_{j}\right)}{N B^{s}} \\
& =\chi\left(A_{i}\right) \sum_{j=1}^{h} \sum_{B \sim A_{i}^{-1} A_{j}} \frac{\chi(B)}{N B^{s}}=\chi\left(A_{i}\right) \cdot L(s, \chi) .
\end{aligned}
$$

Since the determinant is the product of the eigenvalues, we infer using class field theory (see Lang $[6, \mathrm{XII}, \S 1]$ )

COROLlaRY 3.2. $\operatorname{det}\left[\zeta_{\mathcal{A}_{i}^{-1} \mathcal{A}_{j}}(s)\right]=\prod_{\chi} L(s, \chi)=\varsigma_{H}(s)$, where $H$ is the Hilbert class field of $K$.

It remains to find $\operatorname{det}(P)$. First, for an imaginary quadratic field the different is a principal ideal, so that the permutation is actually $A_{i} \rightarrow A_{i}^{-1}$, and therefore $\operatorname{det}(P)=(-1)^{(h-m) / 2}$, where $m$ is the number of elements in the ideal class group of order 2. This, however, can be made more explicit using genus theory (see Hecke $\left[3\right.$, p. 176]), which asserts that $m=2^{t-1}$, where $t$ is the number of prime divisors of $d_{K}$.

Combining Corollaries 2.8 and 3.2 and the above remark concludes the proof of Theorem 1.

We now prove Theorem 2. Let $M=\left(\bar{\chi}_{i}\left(A_{j}\right)\right)_{i, j=1, \ldots, h}$ so that by Proposition 3.1

$$
\left[\zeta_{A_{i}^{-1} A_{j}}(s)\right]=M^{-1}\left[\begin{array}{lll}
L\left(s, \chi_{1}\right) & & \\
& \ddots & \\
& & L\left(s, \chi_{h}\right)
\end{array}\right] M .
$$

Then

$$
\begin{aligned}
& \operatorname{Tr}(\Phi(s))=\operatorname{Tr}\left[\omega_{K}^{2 s-2} \frac{\left(d_{K}^{1 / 2} / 2 \pi\right)^{2-s} \Gamma(2-s)}{\left(d_{K}^{1 / 2} / 2 \pi\right)^{s} \Gamma(s)} \cdot P \cdot\left[\varsigma_{A_{i}^{-1} A_{j}}(2-s)\right]\left[\varsigma_{A_{i}^{-1} A_{j}}(s)\right]^{-1}\right] \\
& =\operatorname{Tr}\left[\omega_{K}^{2 s-2} \frac{\left(d_{K}^{1 / 2} / 2 \pi\right)^{2-s} \Gamma(2-s)}{\left(d_{K}^{1 / 2} / 2 \pi\right)^{s} \Gamma(s)} \cdot P \cdot M^{-1}\left[\begin{array}{lll}
L\left(2-s, \chi_{1}\right) & & \\
& \ddots & \\
& & L\left(2-s, \chi_{h}\right)
\end{array}\right]\right. \\
& \left.\times\left[\begin{array}{lll}
L\left(s, \chi_{1}\right)^{-1} & & \\
& \ddots & \\
& & L\left(s, \chi_{h}\right)^{-1}
\end{array}\right] M\right] .
\end{aligned}
$$

If we assume that $\chi_{1}=1$, then

so that

$$
\lim _{s \rightarrow 1} \frac{L\left(2-s, \chi_{1}\right)}{L\left(s, \chi_{1}\right)}=-1
$$

$$
\begin{aligned}
& \operatorname{Tr}(\Phi(1))=\lim _{s \rightarrow 1} \operatorname{Tr}(\Phi(s))
\end{aligned}
$$

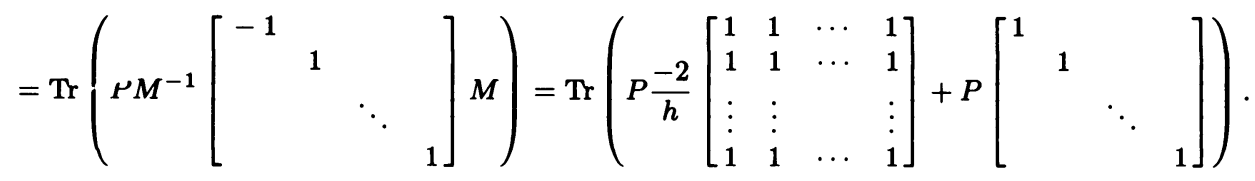


Recalling the definition of $P$ we have $\operatorname{Tr}(\Phi(1))=m-2=2^{t-1}-2$, which is Theorem 2 .

\section{REFERENCES}

1. P. Cohen and P. Sarnak, Discrete groups and geometry (to appear).

2. I. Efrat, The Selberg trace formula for $\mathrm{PSL}_{2}(\mathbf{R})^{n}$, Mem. Amer. Math. Soc. (to appear).

3. E. Hecke, Lectures on the theory of algebraic numbers, Springer-Verlag, 1981.

4. D. Hejhal, The Selberg trace formula for $\mathrm{PSL}_{2}(\mathbf{R})$, vol. 2, Lecture Notes in Math., vol. 1001, Springer-Verlag, 1983.

5. M. Huxley, Scattering matrices for congruence subgroups, preprint, 1983.

6. S. Lang, Algebraic number theory, Addison-Wesley, 1970.

7. P. Lax and R. Phillips, Scattering theory for automorphic forms, Ann. of Math. Studies, no. 87, Princeton Univ. Press, Princeton, N. J., 1976.

8. P. Sarnak, The arithmetic and geometry of some hyperbolic three manifolds, Acta Math. 151 (1983).

9. A. Terras, Harmonic analysis on symmetric spaces with applications to number theory (to appear).

Department of Mathematics, Massachusetts Institute of Technology, CAMBRIDGE, MASSACHUSETTS 02139

Department of Mathematics, Stanford University, Stanford, California 94305 